\title{
Rapid sympathetic cooling to Fermi degeneracy on a chip
}

\author{
S. AUBIN*, S. MYRSKOG, M. H. T. EXTAVOUR, L. J. LEBLANC, D. MCKAY, A. STUMMER AND \\ J. H. THYWISSEN \\ Department of Physics, University of Toronto, Toronto, Ontario M5S 1A7, Canada \\ *e-mail: seth.aubin@utoronto.ca
}

$\mathbf{N}$ eutral fermions present new opportunities for testing models of many-body quantum systems, realizing precision atom interferometry, producing ultra-cold molecules, and investigating fundamental forces. However, since they were first observed ${ }^{1}$, quantum degenerate Fermi gases (DFGs) have continued to be challenging to produce, and have been realized in only a handful of laboratories ${ }^{2-10}$. In this letter, we report the production of a DFG using a simple apparatus based on a microfabricated magnetic trap. Similar approaches applied to Bose-Einstein condensation of ${ }^{87} \mathrm{Rb}$ (refs 11,12) have accelerated evaporative cooling and eliminated the need for multiple vacuum chambers. We demonstrate sympathetic cooling for the first time in a microtrap, and $\operatorname{cool}{ }^{40} \mathrm{~K}$ to Fermi degeneracy in just six seconds - faster than has been possible in conventional magnetic traps. To understand our sympathetic cooling trajectory, we measure the temperature dependence of the ${ }^{40} \mathrm{~K}-{ }^{87} \mathrm{Rb}$ cross-section and observe its RamsauerTownsend reduction.

Microfabricating the electromagnets used to trap ultra-cold atoms leads to a series of experimental benefits. Decreasing the radius $R$ of a surface-mounted wire increases the maximum magnetic field gradient as $R^{-1 / 2}$ (ref. 13). As the oscillation frequency $\omega$ of the trapped atoms increases linearly with transverse field gradient, decreasing $R$ from centimetres to micrometres can increase the confinement frequency by orders of magnitude. In addition, one can imagine a 'lab on a chip', in which multiple devices are integrated on a single device, expediting applications for complex manipulation of fermionic atoms for simulations of strongly correlated systems, quantum transport experiments, collision-insensitive clocks, and precision interferometry ${ }^{14,15}$. The strong confinement provided by a microfabricated electromagnet $(\mu \mathrm{EM})$ trap also has a practical advantage: it facilitates faster cooling, which relaxes constraints on vacuum quality and leads to a tremendous simplification over traditional DFG experiments that require multiple ovens, Zeeman slowers, or two magneto-optical traps (MOTs).

In our system ${ }^{16}$, the entire experimental cycle takes place in a single vapour cell (Fig. 1a). Counter-propagating laser beams collect, cool, and trap $2 \times 10^{7}{ }^{40} \mathrm{~K}$ and $10^{9}{ }^{87} \mathrm{Rb}$ atoms in a MOT. Atoms are transferred to a purely magnetic trap formed by external quadrupole coils and transported to the chip $5 \mathrm{~cm}$ away. Figure $1 \mathrm{~b}$ shows several microscopic gold wires supported by the substrate. In the presence of uniform magnetic fields, current flowing through the central ' $Z$ '-shaped wire creates a magnetic field minimum above the chip. At the centre of this trap, the ${ }^{40} \mathrm{~K}$ radial (longitudinal) oscillation frequency is $\omega_{\perp} / 2 \pi=826 \pm 7 \mathrm{~Hz}\left(\omega_{1} / 2 \pi=46.2 \pm\right.$ $0.7 \mathrm{~Hz}$ ). The corresponding ${ }^{87} \mathrm{Rb}$ trap frequencies are a factor of $\sqrt{m_{\mathrm{Rb}} / m_{\mathrm{K}}} \approx 1.47$ smaller, where $m_{\mathrm{Rb}}$ and $m_{\mathrm{K}}$ are the atomic masses of ${ }^{87} \mathrm{Rb}$ and ${ }^{40} \mathrm{~K}$, respectively.

After loading, the 1.1-mK-deep chip trap holds approximately $2 \times 10^{5}{ }^{40} \mathrm{~K}$ and $2 \times 10^{7}{ }^{87} \mathrm{Rb}$ doubly spin-polarized atoms, at a temperature $\sim 300 \mu \mathrm{K}$. Lower temperatures are achieved by forced evaporative cooling of ${ }^{87} \mathrm{Rb}$. A transverse magnetic field oscillating at radiofrequency (RF) $v_{\mathrm{RF}}$ (typically swept from 30 to $3.61 \mathrm{MHz}$ ) selectively removes the highest energy ${ }^{87} \mathrm{Rb}$ atoms by driving spin-flip transitions to untrapped states. The ${ }^{40} \mathrm{~K}$ atoms, with smaller Zeeman splittings, are not ejected but are sympathetically cooled $\mathrm{d}^{2,17,18}$ by thermalizing with the ${ }^{87} \mathrm{Rb}$ reservoir by means of elastic ${ }^{40} \mathrm{~K}-{ }^{87} \mathrm{Rb}$ collisions $\mathrm{s}^{6,8,10,19}$.

The evolution of temperature $T$ and atom number $N$ during sympathetic cooling is measured by releasing atoms from the trap and observing their expansion with absorptive imaging. Figure 2 shows the cooling of ${ }^{40} \mathrm{~K}$ and ${ }^{87} \mathrm{Rb}$ to quantum degeneracy. In the degenerate regime, bosons accumulate in the ground state (forming a Bose-Einstein condensate), whereas fermions fill the lowest energy levels of the trap with near-unity occupation. Fermi degeneracy can be quantified with the fugacity $\mathbb{Z}$ : the ground state has occupation $\mathbb{Z} /(1+\mathbb{Z})$, which approaches 1 in the high- $\mathbb{Z}$ degenerate limit and $\mathbb{Z}$ in the non-degenerate limit. Owing to the tight confinement of the $\mu \mathrm{EM}$ trap, cooling increases the ${ }^{40} \mathrm{~K}$ fugacity by $10^{12}$ in only $6 \mathrm{~s}$. The steep ascent of fermion fugacity in Fig. 2 also demonstrates the efficiency of sympathetic cooling. The inherent efficiency of sympathetic cooling is significant, as ${ }^{40} \mathrm{~K}$ is a rare isotope, and is therefore more difficult to collect from vapour than ${ }^{87} \mathrm{Rb}$. To our knowledge, this is the first observation of sympathetic cooling, of Fermi degeneracy, and of dual degeneracy in a $\mu$ EM trap.

Below $T \approx 1 \mu \mathrm{K}$, we observe two independent signatures of Fermi degeneracy. First, we compare the r.m.s. cloud size of ${ }^{40} \mathrm{~K}$ and ${ }^{87} \mathrm{Rb}$ (or its non-condensed fraction) by fitting the density 


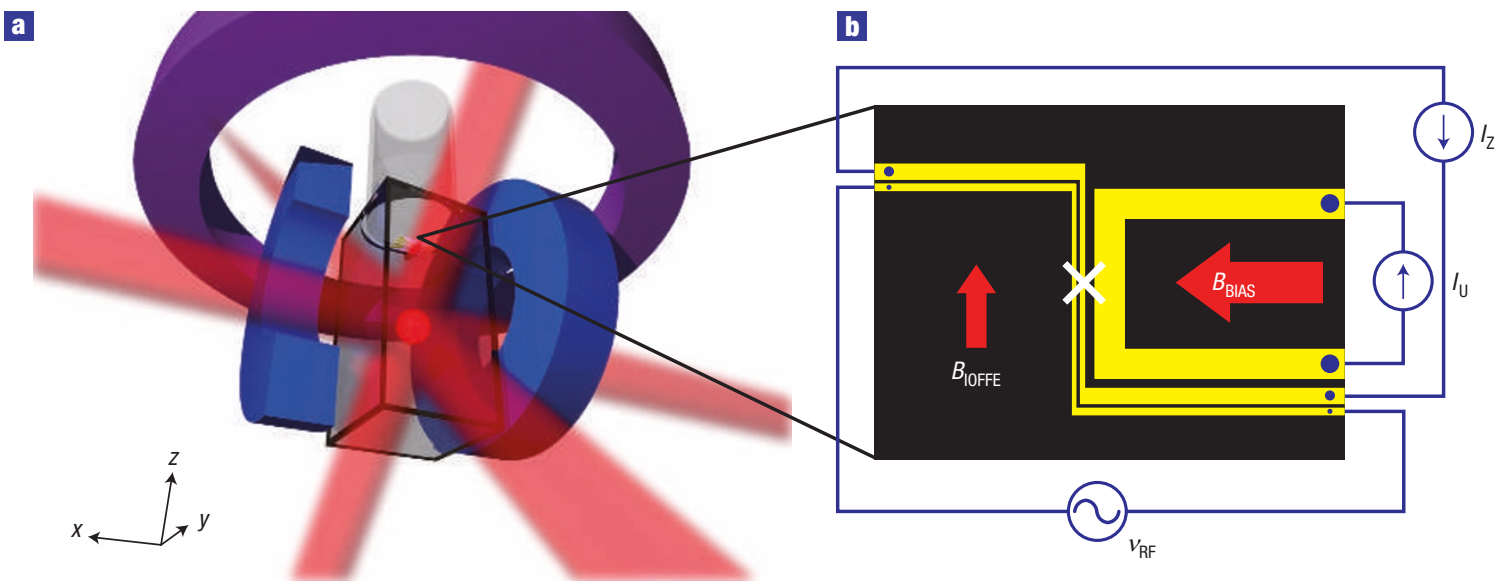

Figure 1 A simple apparatus for Fermi degeneracy. a, The dual-species MOT (red sphere) is formed at the intersection of six laser beams. The cloud is then magnetically trapped using external quadrupole coils (blue), transported $5 \mathrm{~cm}$ vertically using an offset coil (purple), and compressed in the $\mu \mathrm{EM}$ trap. $\mathbf{b}$, Schematic diagram of the central region of the $\mu \mathrm{EM}$ chip. A magnetic trap is formed $180 \mu \mathrm{m}$ above the surface at the location marked with a white ' $\mathrm{X}$ ' by applying $I_{Z}=2.0 \mathrm{~A}, I_{U}=30 \mathrm{~mA}, B_{B \mathrm{BS}}=21.4 \mathrm{G}$, and $B_{\text {loffE }}=5.2 \mathrm{G}$. Wire widths from left to right are 20,60 , and $420 \mu \mathrm{m}$.

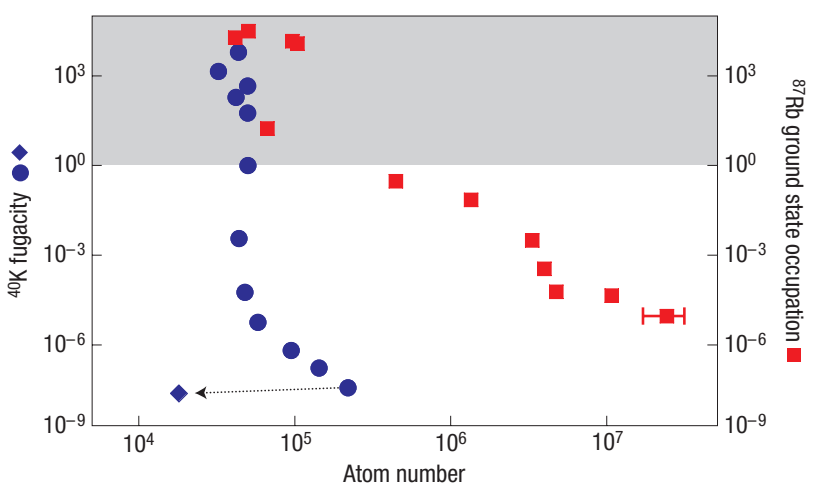

Figure 2 Sympathetic cooling in a chip trap. Spin-polarized fermions without a bosonic bath cannot be successfully evaporatively cooled (blue diamond). However, if bosonic ${ }^{87} \mathrm{Rb}$ (red squares) is evaporatively cooled, then fermionic ${ }^{40} \mathrm{~K}$ is sympathetically cooled (blue dots) to quantum degeneracy (grey area). For bosonic ${ }^{87} \mathrm{Rb}$, the vertical axis is the occupation of the ground state; for fermionic ${ }^{40} \mathrm{~K}$, the vertical axis is the fugacity, as discussed in the text. These two quantities are equivalent in the non-degenerate limit. A typical run-to-run spread in atom number is shown on the right-most point; all vertical error bars are smaller than the marker size.

profiles to a gaussian profile. As described in the Methods section, this is an appropriate method for finding the temperature of a classical Boltzmann gas. Figure 3 shows that the apparent (that is, gaussian-estimated) ${ }^{40} \mathrm{~K}$ temperature approaches a finite value, whereas the ${ }^{87} \mathrm{Rb}$ temperature approaches zero, even though the two gases are in good thermal contact. In fact, this deviation is evidence of the 'Pauli pressure' expected of a gas obeying Fermi statistics ${ }^{2}$ : at zero temperature, fermions fill all available states up to the Fermi energy $E_{\mathrm{F}}=\hbar\left(6 N \omega_{\perp}^{2} \omega_{1}\right)^{1 / 3}$, where $N$ is the number of fermions, and $\hbar$ is the reduced Planck's constant. For our typical parameters, $E_{\mathrm{F}} \approx k_{\mathrm{B}} \times 1.1 \mu \mathrm{K}$. We plot data with thermal and Bose-condensed ${ }^{87} \mathrm{Rb}$ separately, to show that the densitydependent attractive interaction between ${ }^{40} \mathrm{~K}$ and ${ }^{87} \mathrm{Rb}$ does not significantly affect the release energy. A second signature of Fermi statistics is evident in the shape of the cloud. Figure 3, bottom inset, compares the residuals of a gaussian fit (which assumes Boltzmann statistics) with the residuals of a fit which assumes Fermi-Dirac statistics. The Fermi distribution describes the data well, with a $\chi^{2}$ three times lower than the gaussian fit. After all of the ${ }^{87} \mathrm{Rb}$ atoms have been evaporated, we use Fermi-Dirac fits to measure temperature, and find $k_{\mathrm{B}} T / E_{\mathrm{F}}$ as low as $0.09 \pm 0.05$ with as many as $4 \times 10^{4}{ }^{40} \mathrm{~K}$ atoms.

We empirically optimize the sympathetic cooling trajectory, and find that RF sweep times faster than $6 \mathrm{~s}$ are not successful, whereas ${ }^{87} \mathrm{Rb}$ alone can be cooled to degeneracy in $2 \mathrm{~s}$. This indicates that ${ }^{40} \mathrm{~K}$ and ${ }^{87} \mathrm{Rb}$ rethermalize more slowly than ${ }^{87} \mathrm{Rb}$ with itself. Measuring the temperature ratio during sympathetic cooling (Fig. 4a) reveals that ${ }^{40} \mathrm{~K}$ lags behind ${ }^{87} \mathrm{Rb}$ at high temperatures, despite the fact that our optimal frequency ramp starts slowly (when the atoms are hottest), and accelerates at lower temperatures.

In the low-temperature limit, we do not expect the cross-species thermalization to lag the ${ }^{87} \mathrm{Rb}-{ }^{87} \mathrm{Rb}$ thermalization, as the ${ }^{40} \mathrm{~K}-{ }^{87} \mathrm{Rb}$ cross-section $\sigma_{\mathrm{KRb}}=1,480 \pm 70 \mathrm{~nm}^{2}$ (ref. 20) exceeds the ${ }^{87} \mathrm{Rb}-{ }^{87} \mathrm{Rb}$ cross-section, $\sigma_{\mathrm{RbRb}}=689.6 \pm 0.3 \mathrm{~nm}^{2}$ (ref. 21). However, several conflicting values for $\sigma_{\mathrm{KRb}}$ have recently been presented ${ }^{10,19,20,22,23}$.

We investigate $\sigma_{\mathrm{KRb}}$ further by measuring the cross-species thermalization rate $^{24}$ at several temperatures. Starting from equilibrium, we abruptly cool ${ }^{87} \mathrm{Rb}$ by reducing $\nu_{\mathrm{RF}}$, wait for a variable hold time to allow cross-thermalization, and then measure the ${ }^{40} \mathrm{~K}$ temperature, as shown in the inset of Fig. $4 \mathrm{~b}$. We repeat this measurement at several temperatures, and fit each to the model of ref. 25. We find that the cross-section has a dramatic dependence on temperature (see Fig. 4b), decreasing over an order of magnitude between 10 and $200 \mu \mathrm{K}$.

The simplest model for atom-atom scattering uses a deltafunction contact potential. Figure $4 \mathrm{~b}$ shows that the $s$-wave scattering cross-section of this 'naive' model (further described in the Methods section) would predict a higher $\sigma_{\mathrm{KRb}}$ than $\sigma_{\mathrm{RbRb}}$ throughout the cooling cycle, in stark contrast to our measurements. Better agreement is given by an effective-range mode $^{26}$, which includes a reduction in scattering phase (and thus cross-section) below the naive expectation. Our highest temperature data point lies below the effective-range prediction, 


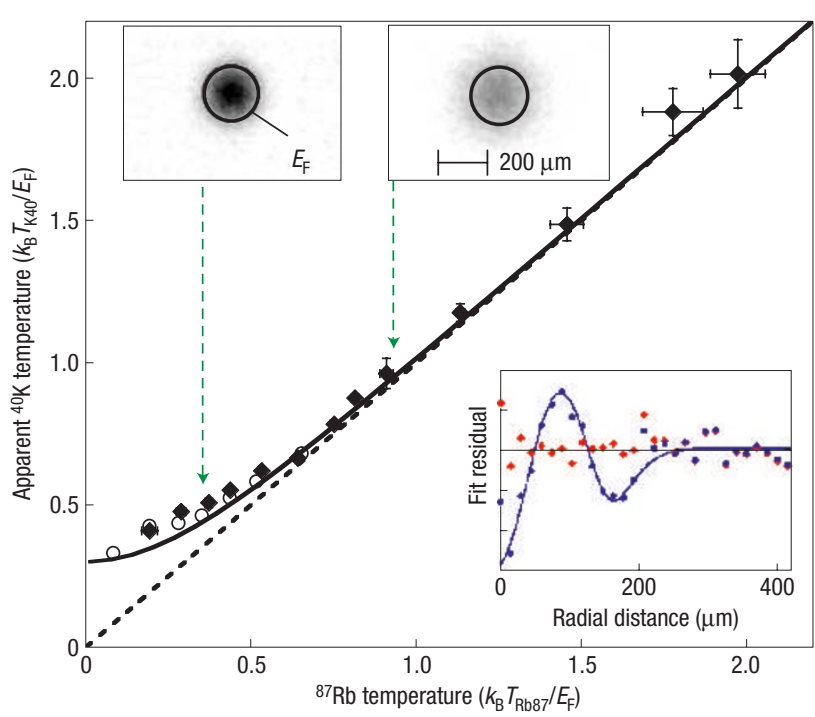

Figure 3 Observation of Fermi statistics. Owing to Pauli pressure, Fermi degenerate ${ }^{40} \mathrm{~K}$ clouds seem to stop getting colder, even when the reservoir temperature approaches zero. The apparent temperature of the fermions, as measured by gaussian fits to images of ${ }^{40} \mathrm{~K}$ clouds, is plotted versus temperature of both thermal (diamonds) and Bose-condensed (circles) ${ }^{87} \mathrm{Rb}$. Data is compared with its classical expectation (dashed line) and with a gaussian fit of theoretically generated ideal Fermi distribution (solid line). Both temperatures are scaled by the Fermi energy $E_{\mathrm{F}}$ of each ${ }^{40} \mathrm{~K}$ cloud. Error bars are statistical (one standard deviation), with uncertainty smaller than the sizes of symbols for lower temperature data. Top insets: Absorption images for $k_{\mathrm{B}} T / E_{\mathrm{F}}=0.35$ (left) and 0.95 (right), including a black circle indicating the Fermi energy $E_{\mathrm{F}}$. Bottom inset: At $k_{\mathrm{B}} T / E_{\mathrm{F}}=0.13_{-0.07}^{+0.04}$ with the ${ }^{87} \mathrm{Rb}$ atoms completely evaporated, a closer look at the fermion cloud shape reveals that it does not follow a Boltzmann distribution. The fit residuals of a radially averaged cloud profile show a strong systematic deviation when assuming Boltzmann (blue circles) instead of Fermi (red diamonds) statistics. A degenerate Fermi cloud is flatter at its centre than a Boltzmann distribution, and falls more sharply to zero near its edge.

however a more-sophisticated analysis may be required to extract a quantitative measurement for this point, due to severe trap anharmonicity at high temperature. Overall, both data and theory show that the ${ }^{40} \mathrm{~K}-{ }^{87} \mathrm{Rb}$ cross-section is reduced well below the ${ }^{87} \mathrm{Rb}-{ }^{87} \mathrm{Rb}$ cross-section for a large range of temperatures, explaining the requirement for a slow initial RF frequency sweep for sympathetic cooling. Below $20 \mu \mathrm{K}$, where no temperature lag is observed, $\sigma_{\mathrm{KRb}}$ exceeds $\sigma_{\mathrm{RbRb}}$.

We attribute the observed reduction in scattering cross-section to the onset of the Ramsauer-Townsend effect, in which the $s$-wave scattering phase and cross-section approach zero for a particular value of relative energies between particles ${ }^{27}$. At higher temperatures, the scattering cross-section should increase again, however free evaporation from our trap limits our measurements to below $300 \mu \mathrm{K}$. Additional partial waves may also affect scattering above the $p$-wave threshold of $110 \mu \mathrm{K}$. Despite the high-temperature reduction in cross-section, ${ }^{40} \mathrm{~K}$ and ${ }^{87} \mathrm{Rb}$ remain relatively good sympathetic cooling partners. For instance, recent measurements of ${ }^{87} \mathrm{Rb}-{ }^{6} \mathrm{Li}$ sympathetic cooling ${ }^{9}$ suggest a zerotemperature cross-section approximately 100 times smaller than $\sigma_{\mathrm{KRb}}$, that is, a maximum cross-section roughly equal to the lowest value we measure here.

The high collision rates in mixtures trapped with a $\mu \mathrm{EM}$ allow us to cool fermions sympathetically to quantum degeneracy in $6 \mathrm{~s}$, faster than previously possible. Our method is an alternative to
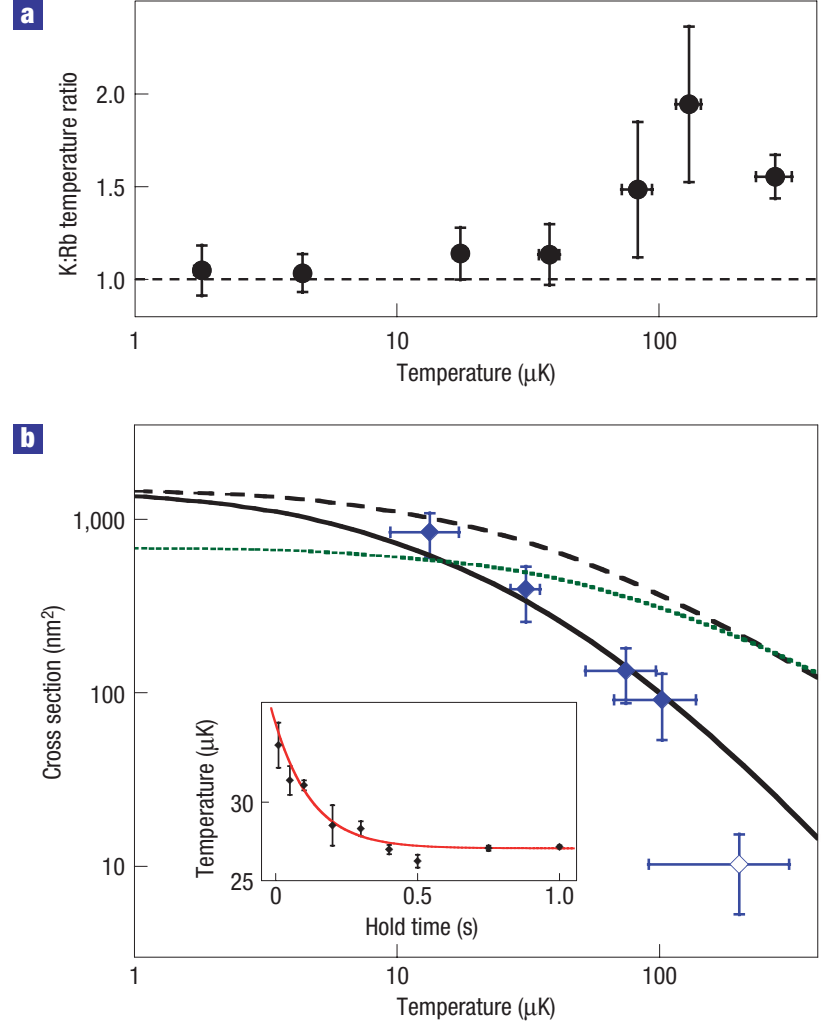

Figure 4 Cross-species thermalization. a, The ratio of the temperature of ${ }^{40} \mathrm{~K}$ to the temperature of ${ }^{87} \mathrm{Rb}$ approaches unity as the ${ }^{40} \mathrm{~K}$ temperature is lowered during sympathetic cooling. $\mathbf{b}$, Measurements of $\sigma_{\mathrm{KRb}}$ (diamonds) are compared with the 'naive' model (dashed) and an effective-range model (solid), both described in the text. Inset: We measure cross-thermalization by abruptly reducing the temperature of ${ }^{87} \mathrm{Rb}$ and watching the temperature of ${ }^{40} \mathrm{~K}$ relax versus time. The data shown has an asymptotic ${ }^{40} \mathrm{~K}$ temperature of $27 \mu \mathrm{K}$. The highest temperature point in $\mathbf{b}$ (open diamond) did not completely thermalize, and is discussed further in the text. For reference, the $s$-wave $\sigma_{\mathrm{RbRb}}$ is also shown (dotted). The error bars shown in all parts of the figure are statistical (one standard deviation), with the exception of the horizontal temperature error bars in $\mathbf{b}$, which show the spread between initial and final ${ }^{40} \mathrm{~K}$ temperature.

all-optical trapping and cooling, which has been used with ${ }^{6} \mathrm{Li}$ to achieve Fermi degeneracy in $3.5 \mathrm{~s}$ (ref. 28). However, magnetic traps allow cooling of fermions without direct evaporative loss, which is critical in the case of ${ }^{40} \mathrm{~K}$ because of its low isotopic abundance. In conclusion, we have achieved simultaneous quantum degeneracy of bosonic and fermionic atoms in a $\mu \mathrm{EM}$ trap and demonstrated an approach that can simplify future research with cold fermions. One prospect is the observation of Pauli blocking in light scattering off degenerate fermions ${ }^{29,30}$. The high $\mu \mathrm{EM}$ trap frequencies boost the ratio of Fermi energy $E_{\mathrm{F}}$ to the recoil energy $\hbar^{2} k^{2} / 2 m_{\mathrm{K}}$ to $\sim 2.5$, within the range necessary to explore such quantum optical effects.

\section{METHODS}

\section{LOADING}

Our experimental cycle is similar to that described in ref. 16, with several key modifications emphasized here and in the main text. Approximately $600 \mathrm{~mW}$ of incoherent 405 -nm light desorbs ${ }^{87} \mathrm{Rb}$ and ${ }^{40} \mathrm{~K}$ atoms from the Pyrex vacuum cell walls, boosting the MOT atom number 100-fold compared with loading from the background vapour. Potassium alone is first loaded into the MOT for $25 \mathrm{~s}$, after which ${ }^{87} \mathrm{Rb}$ is loaded for an additional $3-5 \mathrm{~s}$, while maintaining the 
${ }^{40} \mathrm{~K}$ population. Both MOTs operate with a detuning of $-26 \mathrm{MHz}$, until the last $10 \mathrm{~ms}$, when ${ }^{40} \mathrm{~K}$ is compressed with a $-5 \mathrm{MHz}$ detuning. After MOT loading, 3 ms of optical molasses cooling is applied to the ${ }^{87} \mathrm{Rb}$ atoms, and the ${ }^{40} \mathrm{~K}$ atoms are optically pumped into the $\left|F=9 / 2, m_{\mathrm{F}}=9 / 2\right\rangle$ hyperfine ground state.

\section{MICROELECTROMAGNET TRAP}

$7-\mu \mathrm{m}$-thick gold wires are patterned lithographically and electroplated on a silicon substrate. Two defects are present near the centre of the principal Z-wire, which result in the formation of three 'dimples' in the trapping potential. We use the magnetic gradient generated by $30 \mathrm{~mA}$ of current through the $\mathrm{U}$-wire to centre the magnetic trap on one of these dimples.

\section{FITTING ABSORPTION IMAGE DATA}

Degenerate Fermi clouds are fitted using a semiclassical expression for the optical density: $A f_{2}\left(\mathcal{Z} \exp \left[-\varrho^{2} / 2 r^{2}\right]\right)$, where $\varrho$ is the radial coordinate, $A f_{2}(\mathcal{Z})$ is the peak optical density, $\mathcal{Z}$ the fugacity, and $f_{v}(q)=-\sum_{\ell=1}^{\infty}(-q)^{\ell} / \ell^{v}$ is the Fermi-Dirac function. The temperature is given by $k_{\mathrm{B}} T=r^{2} m_{\mathrm{K}} /\left(\omega_{\perp}^{-2}+t^{2}\right)$, where $r$ is the fit width and $t$ is the time of flight. The atom number is extracted using $N=2 \pi r^{2} f_{3}(\mathcal{Z}) A / \sigma_{\lambda}$, where $\sigma_{\lambda}$ is the resonant absorption cross-section. $T / T_{\mathrm{F}}$ can be extracted directly from the fugacity using $\left(T / T_{\mathrm{F}}\right)^{-3}=6 f_{3}(\mathcal{Z})$. Non-degenerate clouds are fitted to a gaussian distribution $A \exp \left[-\varrho^{2} / 2 r^{2}\right]$, with the same interpretation of $r$. Probes along both $\hat{x}$ and $\hat{y}$ (see Fig. 1) were used for imaging. Comparison of temperature measurements along axes of expansion suggest a $20-\mathrm{nK}$ kick (possibly magnetic) is given to clouds along $\hat{z}$, and that other temperatures agree systematically at the $5 \%$ level. Data for residuals shown in Fig. 3, bottom inset, are radially averaged about an ellipse defined by the two trap frequencies of the image plane. This one-dimensional radial data set is binned into 2-pixel bins, and fitted as described.

\section{SCATTERING THEORIES}

The 'naive' interaction model discussed in the text gives $\sigma_{\mathrm{KRb}}=4 \pi a^{2} /\left(1+a^{2} k^{2}\right)$, where $a$ is the $s$-wave scattering length and $k$ is the relative wave vector in the centre of mass frame. Figure $4 \mathrm{~b}$ shows the thermally averaged theory curves. Including the next-order correction in the $s$-wave scattering amplitude $f(k)=-\left[1 / a+i k+k^{2} r_{\mathrm{e}} / 2+\cdots\right]^{-1}$ requires an effective range, which we calculate using ref. 26 to be $r_{\mathrm{e}}=20.2 \pm 0.3 \mathrm{~nm}$, for $a_{\mathrm{KRb}}=-10.8 \pm 0.3 \mathrm{~nm}$ (ref. 20).

\section{ANALYSIS OF THERMALIZATION DATA}

When the ${ }^{87} \mathrm{Rb}$ atom number $N_{\mathrm{Rb}}$ is much larger than the ${ }^{40} \mathrm{~K}$ atom number, the relaxation of the ${ }^{40} \mathrm{~K}$ temperature $T$ to $T_{\mathrm{Rb}}$ is described by $\dot{u}=-u \tau^{-1}\left(1+m_{\mathrm{Rb}} u /\left(m_{\mathrm{Rb}}+m_{\mathrm{K}}\right)\right)^{1 / 2}(1+u / 2)^{-(3 / 2)}$, where $u \equiv\left(T / T_{\mathrm{Rb}}\right)-1$, and thermalization time $\tau$ given by

$$
\frac{1}{\tau}=\frac{\sqrt{2}}{3 \pi^{2}} \frac{\sigma_{\mathrm{KRb}}}{k_{\mathrm{B}} T_{\mathrm{Rb}}} \frac{\sqrt{m_{\mathrm{K}}} m_{\mathrm{Rb}}^{2} \omega_{\perp}^{2} \omega_{1}}{\left(m_{\mathrm{K}}+m_{\mathrm{Rb}}\right)^{3 / 2}} N_{\mathrm{Rb}},
$$

in which trap frequencies are for ${ }^{87} \mathrm{Rb}$ (ref. 25). Fitting for $\tau$ allows us to extract $\sigma_{\mathrm{KRb}}$. Note that all thermalization data is taken with $N_{\mathrm{K}}$ below $4 \%$ of $N_{\mathrm{Rb}}$.

The data in Fig. $4 \mathrm{~b}$ is analysed assuming a temperature-independent cross-section within the range of initial to final temperature. To check this assumption, we re-analyse the data using a self-consistent method that assumes an effective-range temperature dependence, and find a small upward shift of the best-fit cross-section values. Using this shift as an estimate of the methodology-dependent systematic error, we fit our four lowest temperature measurements with the effective-range model, and find

$a_{\mathrm{KRb}}=-9.9 \pm 1.4 \pm 2.2 \mathrm{~nm}$, in agreement with ref. 20 . The second uncertainty reported is systematic, and also includes uncertainty in the ${ }^{87} \mathrm{Rb}$ number calibration.
Received 20 December 2005; accepted 24 April 2006; published 28 May 2006.

References

1. DeMarco, B. \& Jin, D. S. Onset of Fermi degeneracy in a trapped atomic gas. Science $\mathbf{2 8 5}$, 1703-1706 (1999).

2. Truscott, A. G., Strecker, K. E., McAlexander, W. I., Partridge, G. B. \& Hulet, R. G. Observation of Fermi pressure in a gas of trapped atoms. Science 291, 2570-2572 (2001).

3. Schreck, F. et al. Quasipure Bose-Einstein condensate immersed in a Fermi sea. Phys. Rev. Lett. 87, 080403 (2001).

4. Granade, S. R., Gehm, M. E., O’Hara, K. M. \& Thomas, J. E. All-optical production of a degenerate Fermi gas. Phys. Rev. Lett. 88, 120405 (2002).

5. Hadzibabic, Z. et al. Two-species mixture of quantum degenerate Bose and Fermi gases. Phys. Rev Lett. 88, 160401 (2002)

6. Roati, G., Riboli, F., Modugno, G. \& Inguscio, M. Fermi-Bose quantum degenerate ${ }^{40} \mathrm{~K}-{ }^{87} \mathrm{Rb}$ mixture with attractive interaction. Phys. Rev. Lett. 89, 150403 (2002).

7. Bartenstein, M. et al. Crossover from a molecular Bose-Einstein condensate to a degenerate Fermi gas. Phys. Rev. Lett. 92, 120401 (2004).

8. Köhl, M., Moritz, H., Stöferle, T., Günter, K. \& Esslinger, T. Fermionic atoms in a three dimensional optical lattice: observing Fermi surfaces, dynamics, and interactions. Phys. Rev. Lett. 94, 080403 (2005).

9. Silber, C. et al. Quantum-degenerate mixture of fermionic lithium and bosonic rubidium gases. Phys. Rev. Lett. 95, 170408 (2005).

10. Ospelkaus, C., Ospelkaus, S., Sengstock, K. \& Bongs, K. Interaction-driven dynamics of ${ }^{40} \mathrm{~K} /{ }^{87} \mathrm{Rb}$ Fermi-Bose gas mixtures in the large particle number limit. Phys. Rev. Lett. 96, 020401 (2006).

11. Hänsel, W., Hommelhoff, P., Hänsch, T. W. \& Reichel, J. Bose-Einstein condensation on a microelectronic chip. Nature 413, 498-501 (2001).

12. Ott, H., Fortagh, J., Schlotterbeck, G., Grossmann, A. \& Zimmermann, C. Bose-Einstein condensation in a surface microtrap. Phys. Rev. Lett. 87, 230401 (2001).

13. Groth, S. et al. Atom chips: Fabrication and thermal properties. Appl. Phys. Lett. 85, 2980-2982 (2004)

14. Thywissen, J. H., Westervelt, R. M. \& Prentiss, M. Quantum point contacts for neutral atoms. Phys. Rev. Lett. 83, 3762-3765 (1999).

15. Wang, Y.-J. et al. Atom Michelson interferometer on a chip using a Bose-Einstein condensate. Phys. Rev. Lett. 94, 090405 (2005).

16. Aubin, S. et al. Trapping fermionic ${ }^{40} \mathrm{~K}$ and bosonic ${ }^{87} \mathrm{Rb}$ in a chip trap. J. Low Temp. Phys. 140 $377-396$ (2005)

17. Myatt, C. J., Burt, E. A., Ghrist, R. W., Cornell, E. A. \& Wieman, C. E. Production of two overlapping Bose-Einstein condensates by sympathetic cooling. Phys. Rev. Lett. 78, 586-589 (1997).

18. Schreck, F. et al. Sympathetic cooling of bosonic and fermionic lithium gases towards quantum degeneracy. Phys. Rev. A 64, 011402 (2001).

19. Goldwin, J. et al. Measurement of the interaction strength in a Bose-Fermi mixture with ${ }^{87} \mathrm{Rb}$ and ${ }^{40} \mathrm{~K}$. Phys. Rev. A 70, 021601 (2004).

20. Ferlaino, F. et al. Feschbach spectroscopy of K-Rb atomic mixture. Phys. Rev. A 73, 040702 (2006).

21. van Kempen, E. G. M., Kokkelmans, S. J. J. M. F., Heinzen, J. J. \& Verhaar, B. J. Interisotope determination of ultracold rubidium interactions from three high-precision experiments. Phys. Rev Lett. 88, 93201 (2002).

22. Modugno, G. et al. Collapse of a degenerate Fermi gas. Science 297, 2240-2243 (2002).

23. Inouye, S. et al. Observation of heteronuclear Feshbach resonances in a mixture of bosons and fermions. Phys. Rev. Lett. 93, 183201 (2004).

24. Anderlini, M. et al. Sympathetic cooling and collisional properties of a Rb-Cs mixture. Phys. Rev. A 71, 061401 (2005).

25. Mosk, A. et al. Mixture of ultracold lithium and cesium atoms in an optical dipole trap. Appl. Phys. B 73, 791-799 (2001)

26. Flambaum, V. V., Gribakin, G. F. \& Harabati, C. Analytical calculation of cold-atom scattering. Phys Rev. A 59, 1998-2005 (1999).

27. Townsend, J. S. A Modern Approach to Quantum Mechanics 393 (McGraw-Hill, New York, 1992).

28. O'Hara, K. M. et al. Observation of a strongly interacting degenerate Fermi gas of atoms. Science 298, 2179-2182 (2002).

29. Busch, Th., Anglin, J. R., Cirac, J. I. \& Zoller, P. Inhibition of spontaneous emission in Fermi gases. Europhys. Lett. 44, 1-6 (1998).

30. DeMarco, B. \& Jin, D. S. Exploring a quantum degenerate gas of fermionic atoms. Phys. Rev. A $\mathbf{5 8}$, R4267-R4270 (1998)

\section{Acknowledgements}

We would like to thank D. Jin, J. Dalibard, J. Bohm, and D. Guery-Odelin for helpful conversations about scattering theory, and A. Simoni for sending us unpublished ${ }^{40} \mathrm{~K}-{ }^{87} \mathrm{Rb}$ cross-section calculations. We also thank N. Bigelow, A. Aspect, T. Schumm, and H. Moritz for stimulating conversations, P. Bouyer and R. Nyman for providing a tapered amplifier used in this work, and J. Estève for fabricating the chip used in this work. This work is supported by the NSERC, CFI, OIT, PRO, CRC and Research Corporation. S.A., L.J.L. and D.M. acknowledge support from NSERC. M.H.T.E. acknowledges support from OGS.

Correspondence and requests for materials should be addressed to S.A.

Competing financial interests

The authors declare that they have no competing financial interests.

Reprints and permission information is available online at http://npg.nature.com/reprintsandpermissions/ 